\title{
Conhecimento de acadêmicos de Medicina e recém-formados sobre intubação orotraqueal: um estudo transversal
}

Knowledge of Medical students and recent graduates about orotracheal intubation: a crosssectional study

Conocimiento de estudiantes de Medicina y recién graduados sobre intubación orotraqueal: un estudio transversal

Recebido: 27/10/2021 | Revisado: 05/11/2021 | Aceito: 10/11/2021 | Publicado: 17/11/2021

\author{
Bruno Bibiano de Oliveira \\ ORCID: https://orcid.org/0000-0001-5379-0710 \\ Centro Universitário da Fundação Assis Gurgacz, Brasil \\ E-mail: brunobibiano@hotmail.com \\ Mauro da Cruz Assad Monteiro \\ ORCID: https://orcid.org/0000-0002-3487-7092 \\ Centro Universitário da Fundação Assis Gurgacz, Brasil \\ E-mail: brunobibiano@hotmail.com \\ Rafael Rauber \\ ORCID: https://orcid.org/0000-0002-3231-8836 \\ Centro Universitário da Fundação Assis Gurgacz, Brasil \\ E-mail: rafaelr@fag.edu.br
}

\begin{abstract}
Resumo
A Intubação Orotraqueal (IOT) é um procedimento indicado quando há necessidade do controle definitivo da via aérea. Como a IOT é realizada em situações de emergência, como em paradas cardíacas, existem poucas contraindicações ao procedimento, sendo uma das principais a transecção parcial de traqueia. A chance de sucesso na realização da IOT por estudantes de medicina aumenta em 9\% a cada tentativa (OR 1.09; IC 95\% 1.04 - 1.14) sendo necessárias no mínimo 17 tentativas de IOT para ter $90 \%$ de sucesso no procedimento. Dessa forma, o objetivo deste estudo transversal é avaliar o conhecimento de estudantes de medicina sobre intubação orotraqueal. Observou-se que mais do que $90 \%$ dos respondentes referiram ter conhecimento do por que é realizada IOT, contudo apenas $67,1 \%$ e $53,2 \%$ sabem as indicações e as contraindicações para realização de IOT, respectivamente. 92,4\% dos participantes referiram ter conhecimento da classificação de Mallampati, contudo 82,3\% relataram saber reconhecer uma via aérea difícil de intubar. Verificou-se que médicos recém-formados ou estudantes no internato referem ter mais conhecimento sobre o motivo que leva a realização da IOT $(\mathrm{p}=0,04)$, materiais necessários na IOT $(\mathrm{p}=0,04)$, classificação de Mallampati $(\mathrm{p}<0,01)$ e reconhecimento de via aérea difícil de intubar $(\mathrm{p}=0,04)$ na comparação com acadêmicos cursando ciclo básico ou clínico. Embora seja um estudo limitado por sua metodologia transversal, pode-se observar algumas lacunas no conhecimento, por exemplo apenas 59,5\% dos participantes referiram saber as complicações que podem ser causadas pela IOT.
\end{abstract}

Palavras-chave: Intubação; Educação médica; Competência clínica; Medicina.

\begin{abstract}
Orotracheal intubation (OTI) is a procedure indicated when there is a need for definitive airway control. Since OTI is performed in emergency situations, such as in cardiac arrest, there are few contraindications to the procedure, one of the main ones being partial tracheal transection. The chance of success in performing OTI by medical students increases by $9 \%$ with each attempt (OR 1.09; 95\% CI $1.04-1.14$ ), requiring at least 17 attempts of OTI to have a $90 \%$ success rate. Thus, the aim of this cross-sectional study is to evaluate medical students' knowledge about orotracheal intubation. It was observed that more than $90 \%$ of the respondents reported knowing why OTI is performed; however, only $67.1 \%$ and $53.2 \%$ know the indications and contraindications for performing OTI, respectively. $92.4 \%$ of the participants reported knowing about Mallampati's classification, however 82.3\% reported that they know how to recognize an airway that is difficult to intubate. It was found that recently graduated or internship students reported having more knowledge about the reason to perform OTI ( $\mathrm{p}=0.04)$, materials needed for OTI $(\mathrm{p}=0.04)$, Mallampati classification $(\mathrm{p}<0.01)$ and recognition of a difficult to intubate airway $(\mathrm{p}=0.04)$ when compared to students in basic or clinical stage. Although it is a limited study due to its cross-sectional methodology, some gaps in knowledge can be observed, for example only $59.5 \%$ of the participants reported knowing the complications that can be caused by OTI.
\end{abstract}

Keywords: Intubation; Medical education; Clinical competence; Medicine. 


\begin{abstract}
Resumen
La intubación orotraqueal (IOT) es un procedimiento indicado cuando es necesario el control definitivo de la vía aérea. Dado que la IOT se realiza en situaciones de emergencia, como en una parada cardíaca, existen pocas contraindicaciones para el procedimiento, siendo una de las principales la transección traqueal parcial. La probabilidad de éxito en la realización de la IOT por parte de los estudiantes de medicina aumenta 9\% con cada intento (OR 1,09; IC 95\%: 1,04 $1,14)$, siendo necesarios al menos 17 intentos de IOT para tener $90 \%$ de éxito en el procedimiento. Así pues, el objetivo de este estudio transversal era evaluar los conocimientos de los estudiantes de medicina sobre la intubación orotraqueal. Se observó que más del $90 \%$ de los encuestados dijeron tener conocimientos sobre por qué se realiza la IOT, sin embargo, sólo $67,1 \%$ y 53,2\% conocen las indicaciones y contraindicaciones para realizar la IOT, respectivamente. 92,4\% de los participantes dijo tener conocimientos sobre la clasificación de Mallampati, sin embargo 82,3\% dijo saber reconocer una vía aérea difícil de intubar. Se observó que los estudiantes recién graduados o en prácticas informaron que tenían más conocimientos sobre el motivo para realizar la IOT $(\mathrm{p}=0,04)$, los materiales necesarios en la IOT $(\mathrm{p}=0,04)$, la clasificación de Mallampati $(\mathrm{p}<0,01)$ y el reconocimiento de las vías aéreas difíciles de intubar $(\mathrm{p}=0,04)$ en comparación con los estudiantes de ciclo básico o clínico. Aunque se trata de un estudio limitado debido a su metodología transversal, se observan algunas lagunas en los conocimientos, por ejemplo, sólo 59,5\% de los participantes declaró conocer las complicaciones que puede provocar la IOT.
\end{abstract}

Palabras clave: Intubación; Educación médica; Competencia clínica; Medicina.

\title{
1. Introdução
}

A Intubação Orotraqueal (IOT) é um procedimento indicado quando há necessidade do controle definitivo da via aérea. Na emergência, é realizada nos casos de falência respiratória ou cardíaca, falha de proteger a via aérea de aspiração, ventilação ou oxigenação inadequada ou obstrução aérea (Kabrhel et al 2007).

Como a IOT é realizada em situações de emergência, como em paradas cardíacas, existem poucas contraindicações ao procedimento, sendo uma das principais a transecção parcial de traqueia pois o procedimento pode causar uma transecção completa, sendo recomendado a confecção de uma via cirúrgica em detrimento da IOT (Kabrhel et al 2007).

Os equipamentos necessários para realização da IOT são luvas, protetor facial, sistema de sucção, bolsa-valva-máscara ligada a fonte de oxigênio, tubo endotraqueal, seringa, estetoscópio e laringoscópio (Kabrhel et al 2007). De acordo com Higgs et al (2017) a técnica de IOT consiste das seguintes etapas: preparação, onde são preparados os equipamentos e drogas necessárias, na pré-oxigenação, que é necessária devido ao período de apneia associada ao bloqueio neuromuscular, levando a hipoxemia. Após a realização da pré-oxigenação pode-se administrar um agente de indução da anestesia, como a Cetamina, juntamente com um bloqueador neuromuscular, como a succinilcolina ou rocurônio. Após a administração das drogas, o profissional deve visualizar, com o auxílio do laringoscópio a via aérea e posicionar o tubo endotraqueal na laringe. Para confirmar a correta localização do tubo pode ser realizada uma capnografia e radiografia de tórax.

Em estudo realizado por Tarasi et al (2011) observou-se que a chance de sucesso na realização da intubação orotraqueal (IOT) por estudantes de medicina aumenta em 9\% a cada tentativa (OR 1.09; IC 95\% 1.04 - 1.14) e foram necessários no mínimo 17 tentativas de IOT para ter $90 \%$ de sucesso no procedimento. Taxas de sucesso na IOT são reduzidas na primeira tentativa realizada por estudantes de medicina, pois apenas $33 \%$ realizaram a intubação corretamente e $47 \%$ identificaram incorretamente o posicionamento do tubo endotraqueal (O’Flaherty \& Adams, 1992).

Estudos da literatura demonstram que $71 \%$, 95\% e $81 \%$ dos acadêmicos nunca realizaram acesso arterial, acesso intraósseo e acesso venoso central, respectivamente (Barr \& Graffeo, 2016). Dessa forma, existe uma baixa taxa de realização de procedimentos médicos em geral por parte dos estudantes de medicina.

Portanto, é essencial que médicos recém-formados e estudantes de medicina concluindo o ciclo de internato estejam aptos a indicar e saber realizar procedimentos médicos, como a intubação orotraqueal. Contudo, estudos da literatura demonstram que $33 \%$ dos estudantes de medicina nos Estados Unidos nunca realizaram uma IOT (Barr \& Graffeo, 2016). Como não foram encontradas estatísticas brasileiras a respeito deste tema, este estudo buscou avaliar o conhecimento sobre intubação orotraqueal 
de estudantes de medicina nos ciclos básico, clínico e estágios obrigatórios (internato) e médicos recém-formados para verificar qual o nível de domínio dos participantes referente a este importante procedimento médico.

\section{Metodologia}

Trata-se de um estudo quantitativo observacional com abordagem longitudinal, realizada através do preenchimento de um questionário. Setenta e nove estudantes de medicina ou médicos recém-formados ( $<3$ anos desde a formatura) participaram do estudo após concordarem com o Termo de Consentimento Livre e Esclarecido. A pesquisa foi aprovada pelo Comitê de Ética em Pesquisas Com Seres Humanos do Centro Universitário da Fundação Assis Gurgacz com número CAAE 50232421.1.0000.5219. A base metodológica utilizada no estudo foi estruturada pelo trabalho de Pereira et al (2018).

Os critérios de inclusão foram idade $\geq 18$ anos e estar matriculado no curso de medicina ou ser médico recém-formado. Desta forma, foram excluídos do estudo quem se recusou a aceitar o Termo de Consentimento Livre e Esclarecido, participantes que preencheram parcialmente o formulário, estudantes e graduados de outros cursos, participantes com idade < 18 anos e médicos que se formaram a mais do que 3 anos.

As análises estatísticas foram realizadas no software RStudio na versão 1.4.1103 utilizando teste de Mann-Whitney (Tabela 2) e Fisher (Tabela 3). Para a associação estatística ser considerada válida foi utilizado nível de significância ( $\alpha$ ) de 0,05 , sendo consideradas estatisticamente relevantes associações cujo p-valor foi inferior a 0,05 .

\section{Resultados e Discussão}

Na Tabela 1 foram descritas características dos participantes da pesquisa. Com relação a etapa de formação, 8,9\% estavam no ciclo básico, 35,4\% no ciclo clínico, $41,8 \%$ no internato e 13,9\% eram médicos recém-formados. Cerca de $65 \%$ dos participantes julga como mediano (4-7) seu conhecimento sobre Intubação Orotraqueal (IOT) e 19\% julgam como bom/ótimo. Mais do que $90 \%$ dos respondentes referiram ter conhecimento do por que é realizada IOT, contudo apenas $67,1 \%$ e $53,2 \%$ sabem as indicações e as contraindicações para realização de IOT, respectivamente. Com relação aos materiais necessários na IOT, $82,3 \%$ responderam que sabem quais são.

As classificações de Mallampati e Cormack e Lehane são utilizadas para prever a dificuldade de intubação de um paciente (Mallampati et al, 1985; Cormack e Lehane, 1984). 92,4\% dos participantes referiram ter conhecimento da classificação de Mallampati, contudo apenas 21,5\% sabem sobre a classificação de Cormack e Lehane. 82,3\% relataram saber reconhecer uma via aérea difícil de intubar. Estudos da literatura sugerem que vias aéreas difíceis de intubar apresentam maiores chances de complicações (De Jong et al, 2015; Wang et al, 2015), como intubação esofágica, hipoxemia, hipotensão severa (Griesdale et al, 2008; Rashkin \& Davis, 1986) e injúrias laríngeas (Mota, Carvalho e Brito, 2012), contudo apenas 59,5\% dos entrevistados responderam que sabem as principais complicações da IOT.

Visando ampliar o conhecimento dos estudantes de medicina sobre IOT, diversos estudos buscaram avaliar metodologias mais eficazes de ensino do procedimento. Em estudo realizado por Danek, Arruda e Quilici (2016) acadêmicos que estudaram por meio de materiais em vídeo tiveram melhor desempenho na avaliação teórica ( $\mathrm{p}<0,001)$ na comparação com estudantes que utilizaram checklists, contudo a diferença não foi estatisticamente relevante na execução do procedimento $(\mathrm{p}=1)$. Além disso, outro estudo demonstrou que o método de 4 passos de Payton, que consistem em demonstração, desconstrução, compreensão e execução foi superior ao método padrão de aprendizagem $(\mathrm{p}<0,001)$ (Zamani et al, 2020). 
Tabela 1: Descrição das características dos participantes da pesquisa sobre Intubação Orotraqueal (IOT) $(n=79)$.

\begin{tabular}{lcc}
\hline Característica & $\mathbf{n}$ & $\%$ \\
\hline Etapa de formação & & \\
Ciclo Básico & 7 & $8,9 \%$ \\
Ciclo Clínico & 28 & $35,4 \%$ \\
Internato & 33 & $41,8 \%$ \\
Médico recém-formado & 11 & $13,9 \%$
\end{tabular}

Em uma escala de 0 a 10, como julga seu conhecimento sobre IOT?

$\begin{array}{lcc}0-3 & 13 & 16,5 \% \\ 4-7 & 51 & 64,6 \% \\ 8-10 & 15 & 19 \%\end{array}$

Tem conhecimento do por que é realizada IOT?

$\begin{array}{lcc}\text { Sim } & 72 & 91,1 \% \\ \text { Não } & 7 & 8,9 \% \\ \text { m conhecimento sobre indicações de IOT? } & \\ \text { Sim } & 53 & 67,1 \% \\ \text { Não } & 26 & 32,9 \%\end{array}$

Tem conhecimento sobre contraindicações de IOT?

$\begin{array}{lll}\text { Sim } & 42 & 53,2 \% \\ \text { Não } & 37 & 46,8 \%\end{array}$

Tem conhecimento sobre materiais necessários na IOT?

$\begin{array}{lll}\text { Sim } & 65 & 82,3 \% \\ \text { Não } & 14 & 17,7 \%\end{array}$

Conhecimento sobre a fisiologia da necessidade de hiperventilação antes da IOT?

$\begin{array}{lll}\text { Sim } & 65 & 82,3 \% \\ N \tilde{a} o & 14 & 17,7 \%\end{array}$

$17,7 \%$

Conhece classificação de Mallampati?

Sim $\quad 73 \quad 92,4 \%$

Não $\quad 6 \quad 7,6 \%$

Conhece classificação de Cormack e Lehane?

$\begin{array}{lll}\text { Sim } & 17 & 21,5 \% \\ \text { Não } & 62 & 78,5 \%\end{array}$

Sabe reconhecer uma via aérea difícil de intubar?

$\begin{array}{lll}\text { Sim } & 65 & 82,3 \% \\ \text { Não } & 14 & 17,7 \%\end{array}$

Conhece as possíveis complicações da IOT?

Sim $47 \quad 59,5 \%$

Não $\quad 32 \quad 40,5 \%$

Fonte: Autores (2021).

Na Tabela 2 foi avaliado a pontuação média na autoavaliação do conhecimento sobre IOT em uma escala de 0 a 10. Embora a pontuação média foi de 5,93 nos médicos recém-formados e estudantes cursando internato e de 5,34 pontos para acadêmicos do ciclo básico ou clínico a diferença entre os grupos não foi estatisticamente relevante $(\mathrm{p}=0,45)$. 
Tabela 2: Pontuação média da autoavaliação do conhecimento sobre Intubação Orotraqueal (IOT) em estudantes de medicina e médicos recém-formados

\begin{tabular}{lcc}
\hline Etapa de formação & $\begin{array}{c}\text { Pontuação média } \\
\pm \mathbf{D P}\end{array}$ & $\mathbf{p}$ \\
\hline $\begin{array}{l}\text { Ciclo Básico ou Clínico } \\
\begin{array}{l}\text { Internato ou recém- } \\
\text { formado }\end{array}\end{array}$ & $5,34 \pm 2,54$ & $0,45^{1}$ \\
\hline
\end{tabular}

${ }^{1}$ Teste de Mann-Whitney. Fonte: Autores (2021).

Na Tabela 3 foi demonstrado a associação entre conhecimento sobre IOT com etapa de formação. Observou-se estudantes cursando o internato ou médicos recém-formados referiram, com relevância estatística, ter mais conhecimentos do por que é realizada IOT $(\mathrm{p}=0,04)$, sobre materiais necessários na IOT $(\mathrm{p}=0,04)$, sobre classificação de Mallampati ( $\mathrm{p}<0,01)$ e referente ao reconhecimento de vias aéreas difíceis de intubar $(\mathrm{p}=0,04)$ na comparação com acadêmicos do ciclo básico ou clínico. Contudo, estes estudantes referiram proporcionalmente mais conhecimento sobre a classificação de Cormack e Lehane ( $\mathrm{p}=0,03)$ na comparação com acadêmicos do internato e médicos recém-formados.

Tabela 3: Associação do conhecimento sobre Intubação Orotraqueal (IOT) dos estudantes de medicina e médicos recém-formados com relação a etapa de formação.

\begin{tabular}{|c|c|c|c|c|}
\hline Pergunta & $\operatorname{Sim}(\%)$ & Não $(\%)$ & $\begin{array}{c}\text { Razão de } \\
\text { Prevalências } \\
\text { (IC 95\%) }\end{array}$ & $\mathbf{p}^{1}$ \\
\hline \multicolumn{5}{|c|}{ Conhecimento do por que é realizada IOT } \\
\hline Ciclo Básico ou Clínico & $29(82,9 \%)$ & $6(17,1 \%)$ & 1 & \\
\hline Internato ou recém-formado & $43(97,7 \%)$ & $1(2,3 \%)$ & $\begin{array}{c}1,18 \\
(1,01-1,38)\end{array}$ & 0,04 \\
\hline \multicolumn{5}{|c|}{ Conhecimento sobre indicações de IOT } \\
\hline Ciclo Básico ou Clínico & $20(57,1 \%)$ & $15(42,9 \%)$ & 1 & \\
\hline Internato ou recém-formado & $33(75 \%)$ & $11(25 \%)$ & $\begin{array}{c}1,31 \\
(0,94-1,83)\end{array}$ & 0,15 \\
\hline \multicolumn{5}{|c|}{ Conhecimento sobre contraindicações de IOT } \\
\hline Ciclo Básico ou Clínico & $15(42,9 \%)$ & $20(57,1 \%)$ & 1 & \\
\hline Internato ou recém-formado & $27(61,4 \%)$ & $17(38,6 \%)$ & $\begin{array}{c}1,43 \\
(0,91-2,24)\end{array}$ & 0,12 \\
\hline \multicolumn{5}{|c|}{ Conhecimento sobre materiais necessários na IOT } \\
\hline Ciclo Básico ou Clínico & $25(71,4 \%)$ & $10(28,6 \%)$ & 1 & \\
\hline Internato ou recém-formado & $40(90,9 \%)$ & $4(9,1 \%)$ & $\begin{array}{c}1,27 \\
(1,01-1,60)\end{array}$ & 0,04 \\
\hline \multicolumn{5}{|c|}{ Conhecimento sobre a fisiologia da necessidade de hiperventilação antes da IOT? } \\
\hline Ciclo Básico ou Clínico & $26(74,3 \%)$ & $9(25,7 \%)$ & 1 & \\
\hline Internato ou recém-formado & $39(88,6 \%)$ & $5(11,4 \%)$ & $\begin{array}{c}1,19 \\
(0,96-1,49)\end{array}$ & 0,14 \\
\hline \multicolumn{5}{|c|}{ Conhecimento sobre classificação de Mallampati } \\
\hline Ciclo Básico ou Clínico & $29(82,9 \%)$ & $6(17,1 \%)$ & 1 & \\
\hline Internato ou recém-formado & $44(100 \%)$ & $0(0 \%)$ & $\begin{array}{c}1,21 \\
(1,03-1,41)^{2}\end{array}$ & $<0,01^{2}$ \\
\hline \multicolumn{5}{|c|}{ Conhecimento sobre classificação de Cormack e Lehane } \\
\hline Ciclo Básico ou Clínico & $12(34,3 \%)$ & $23(65,7 \%)$ & $\begin{array}{c}3,02 \\
(1,17-7,76)\end{array}$ & 0,03 \\
\hline Internato ou recém-formado & $5(11,4 \%)$ & $39(88,6 \%)$ & 1 & \\
\hline \multicolumn{5}{|c|}{ Reconhecimento de via aérea difícil de intubar } \\
\hline Ciclo Básico ои Clínico & $25(71,4 \%)$ & $10(28,6 \%)$ & 1 & \\
\hline Internato ou recém-formado & $40(90,9 \%)$ & $4(9,1 \%)$ & $\begin{array}{c}1,27 \\
(1,01-1,60)\end{array}$ & 0,04 \\
\hline \multicolumn{5}{|c|}{ Conhecimento sobre as possíveis complicações da IOT? } \\
\hline Ciclo Básico ou Clínico & $21(60 \%)$ & $14(40 \%)$ & 1 & \\
\hline Internato ou recém-formado & $26(59,1 \%)$ & $18(40,9 \%)$ & $\begin{array}{c}0,98 \\
(0,68-1,42)\end{array}$ & 1 \\
\hline
\end{tabular}

Fonte: Autores (2021) 
Embora médicos recém-formados ou estudantes cursando o internato referiram proporcionalmente mais conhecimentos referente as indicações de IOT (RP 1,31; IC 95\% 0,94 - 1,83), contraindicações de IOT (RP 1,43; 0,91 - 2,24) e fisiologia da necessidade de hiperventilação antes da IOT (RP 1,19; IC 95\% 0,96 - 1,49) a diferença de conhecimento nestas questões não foi estatisticamente relevante (Tabela 3).

\section{Conclusão}

Observou-se neste estudo que houve progressão do conhecimento referido por participantes no internato ou recémformados em algumas das questões relacionadas a IOT, como o motivo que leva a realização da IOT (p=0,04), materiais necessários na IOT $(\mathrm{p}=0,04)$, classificação de Mallampati $(\mathrm{p}<0,01)$ e reconhecimento de via aérea difícil de intubar ( $\mathrm{p}=0,04)$ na comparação com acadêmicos cursando ciclo básico ou clínico. Contudo, não houve progressão estatisticamente relevante na pontuação média da autoavaliação geral sobre conhecimento de questões relacionadas a IOT ( $\mathrm{p}=0,45)$, indicações de IOT $(\mathrm{p}=0,15)$, contraindicações de IOT $(\mathrm{p}=0,12)$ e fisiologia da necessidade de hiperventilação antes da IOT ( $\mathrm{p}=14)$ e possíveis complicações da IOT $(\mathrm{p}=1)$ (Tabela 2 e 3$)$.

Encontrou-se neste estudo lacunas no conhecimento de tópicos referentes a IOT. Por exemplo, apenas 59,5\% dos participantes referiram saber as complicações que podem ser causadas pela IOT (Tabela 1). Portanto, é essencial que sejam aplicadas metodologias baseadas em evidências que sejam eficazes no aprendizado dos acadêmicos além da possibilidade de realização do procedimento na prática, pois apenas após a realização de 17 IOTs é possível predizer uma taxa de sucesso de $90 \%$ (Tarasi et al, 2011).

Futuros estudos podem abordar a temática do conhecimento sobre IOT em estudantes de medicina e médicos recémformados utilizando uma abordagem longitudinal, onde os acadêmicos realizam um teste sobre IOT em cada uma das etapas de formação (ciclo básico, clínico, internato e após formatura) para avaliar a progressão do conhecimento em cada uma das fases.

\section{Referências}

Barr, J., \& Graffeo, C. S. (2016). Procedural Experience and Confidence Among Graduating Medical Students. Journal of Surgical Education, 73(3), 466-473. https://doi.org/10.1016/j.jsurg.2015.11.014

Cormack, R. S., \& Lehane, J. (1984). Difficult tracheal intubation in obstetrics. Anaesthesia, 39(11), 1105-1111. http://www.ncbi.nlm.nih.gov/pubmed/6507827

Danek, A., Arruda, F. T. de, \& Quilici, A. P. (2016). Comparação da Eficiência do Treinamento em Entubação Orotraqueal com Vídeo Educacional versus Checklist. Revista Brasileira de Educação Médica, 40(4), 560-564. https://doi.org/10.1590/1981-52712015v40n4e01592014

De Jong, A., Molinari, N., Pouzeratte, Y., Verzilli, D., Chanques, G., Jung, B., Futier, E., Perrigault, P.-F., Colson, P., Capdevila, X., \& Jaber, S. (2015). Difficult intubation in obese patients: incidence, risk factors, and complications in the operating theatre and in intensive care units. British Journal of Anaesthesia, 114(2), 297-306. https://doi.org/10.1093/bja/aeu373

Griesdale, D. E. G., Bosma, T. L., Kurth, T., Isac, G., \& Chittock, D. R. (2008). Complications of endotracheal intubation in the critically ill. Intensive Care Medicine, 34(10), 1835-1842. https://doi.org/10.1007/s00134-008-1205-6

Kabrhel, C., Thomsen, T. W., Setnik, G. S., \& Walls, R. M. (2007). Orotracheal Intubation. New England Journal of Medicine, 356(17), e15. https://doi.org/10.1056/NEJMvcm063574

Mallampati, S. R., Gatt, S. P., Gugino, L. D., Desai, S. P., Waraksa, B., Freiberger, D., \& Liu, P. L. (1985). A clinical sign to predict difficult tracheal intubation; a prospective study. Canadian Anaesthetists' Society Journal, 32(4), 429-434. https://doi.org/10.1007/BF03011357

Mota, L., de Cavalho, G., \& Brito, V. (2012). Laryngeal complications by orotracheal intubation: Literature review. International Archives of Otorhinolaryngology, 16(02), 236-245. https://doi.org/10.7162/S1809-97772012000200014

O'Flaherty, D., \& Adams, A. P. (1992). Endotracheal intubation skills of medical students. Journal of the Royal Society of Medicine, 85(10), 603-604. http://www.ncbi.nlm.nih.gov/pubmed/1433035

Pereira A. S. et al. (2018). Metodologia da pesquisa científica. UFSM

Rashkin, M. C., \& Davis, T. (1986). Acute Complications of Endotracheal Intubation. Chest, 89(2), 165-167. https://doi.org/10.1378/chest.89.2.165

Tarasi MD, P. G., Mangione MD, M. P., Singhal, S. S., \& Wang MD MS, H. E. (2011). Endotracheal intubation skill acquisition by medical students. Medical Education Online, 16(1), 7309. https://doi.org/10.3402/meo.v16i0.7309 
Research, Society and Development, v. 10, n. 15, e37101522299, 2021

(CC BY 4.0) | ISSN 2525-3409 | DOI: http://dx.doi.org/10.33448/rsd-v10i15.22299

Wang, C. M. Z., Pang, K. P., Tan, S. G., Pang, K. A., Pang, E. B., Cherilynn, T. Y. N., Chan, Y. H., \& Rotenberg, B. W. (2019). Predictors of difficulty in intubation in patients with obstructive sleep apnoea. The Medical Journal of Malaysia, 74(2), 133-137. http://www.ncbi.nlm.nih.gov/pubmed/31079124

Zamani, M., Nasr-Esfahani, M., Forghani, M., Sichani, M., \& Omid, A. (2020). Endotracheal intubation training to medical practitioners: Comparison of the modified 4-step Payton's training method and Halsted's training method in a simulated environment. Journal of Education and Health Promotion, 9(1), 126. https://doi.org/10.4103/jehp.jehp_705_19 